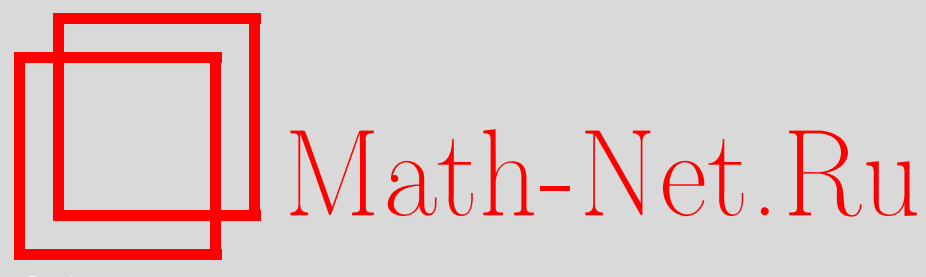

В. В. Пупышев, Строение регулярных решений уравнений Фаддеева вблизи точки парного удара, ТМФ, 2008, том 156, номер 1, 112-130

DOI: https://doi.org/10.4213/tmf6233

Использование Общероссийского математического портала Math-Net.Ru подразумевает, что вы прочитали и согласны с пользовательским соглашением http://www . mathnet.ru/rus/agreement

Параметры загрузки:

IP : 54.84 .234 .179

26 апреля 2023 г., 13:51:39

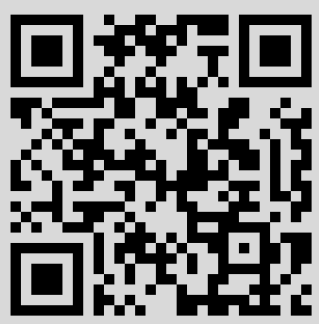




\section{СТРОЕНИЕ РЕГУЛЯРНЫХ РЕШЕНИЙ УРАВНЕНИЙ ФАДДЕЕВА ВБЛИЗИ ТОЧКИ ПАРНОГО УДАРА}

Исследуются двумерные и трехмерные уравнения Фаддеева для системы трех частиц с центральными или $S$-волновыми парными взаимодействиями. Регулярные решения таких уравнений представлены бесконечными рядами по целым степеням расстояния между двумя частицами и искомым функциям других трехчастичных координат. Построение таких функций сведено к решению алгебраических рекуррентных уравнений. Для регулярных решений уравнений Фаддеева выведены граничные условия в точке парного удара.

Ключевые слова: задача трех частиц, дифференциальные уравнения Фаддеева, регулярные решения, точка парного удара.

\section{1. ВВЕДЕНИЕ}

После работы Като [1] разложения трехчастичной волновой функции вблизи точки парного удара в случае кулоновских парных взаимодействий исследовались многими авторами. Результаты таких исследований представлены в работах [2]-[6] и во многих других работах, обсужденных в обзоре [7]. Наиболее полный анализ строения волновой функции вблизи точки парного удара дан в работе [8], где исследовалось шестимерное уравнение Шредингера для системы трех частиц с центральными парными взаимодействиями более общего вида, чем кулоновские. Регулярные общее и частные физические решения такого уравнения были представлены бесконечными рядами по целым степеням расстояния между двумя частицами и искомым функциям других трехчастичных координат. Построение таких функций в угловых базисах, образованных сферическими и бисферическими гармониками или симметризованными $D$-функциями Вигнера, было сведено к решению простых алгебраических рекуррентных уравнений. Для проекций физических решений на угловые базисные функции были выведены граничные условия в точке парного удара.

Настоящая работа является продолжением вышеупомянутой работы [8] и посвящена построению и анализу разложений регулярных решений двумерных и трехмерных уравнений Фаддеева [9]-[11] вблизи точки парного удара. Главная цель

* Объединенный институт ядерных исследований, г. Дубна, Московская обл., Россия. E-mail: pupyshev@thsun1.jinr.ru 
нашего анализа - вывод связей в точке парного удара, т.е. линейных соотношений, связывающих частные производные исследуемых решений в этой точке.

$\mathrm{C}$ теоретической точки зрения искомые связи интересны как аналоги и обобщения давно известного условия Като, но уже для парциальных фаддеевских компонент волновой функции. Вывод таких связей представляется актуальным и с практической точки зрения. Дело в том, что включение связей в дискретные сплайн-аналоги [12], [13] уравнений Фаддеева - простой способ заметно улучшить поточечную сходимость вычисляемого решения к точному вблизи точки парного удара.

Наши исследования основаны на известном в теории дифференциальных уравнений асимптотическом методе [14], [15], когда решение исследуемого дифференциального уравнения в частных производных ищется в виде бесконечного степенного ряда по одному аргументу и неизвестным функциям всех других аргументов. На первом этапе для таких функций из исходного уравнения выводится рекуррентная цепочка бесконечных систем уравнений, а затем доказывается ее однозначная разрешимость. Если описанный этап удается завершить, то построенный ряд называется формальным асимптотическим решением исходного дифференциального уравнения. Следующий и, как правило, довольно сложный этап состоит в доказательстве асимптотической сходимости и дифференцируемости полученного ряда.

В настоящей работе реализуется первый этап построения асимптотических разложений регулярных решений двумерных и трехмерных уравнений Фаддеева вблизи точки парного удара: для таких решений выводятся формальные асимптотические разложения, вопрос о сходимости которых в каком-либо смысле остается открытым. Доказательство сходимости принципиально затрудняется наличием в полученных разложениях произвольных функций. Поэтому предполагается, что все разложения асимптотические и дифференцируемые, а все содержащиеся в них неопределенные функции отличны от нуля. Далее выводятся явные асимптотики полученных рядов в виде подсумм их нескольких наиболее медленно убывающих слагаемых. Наконец, вычисляются частные производные разных порядков таких подсумм в точке парного удара и из полученной совокупности выражений исключаются все неопределенные функции. В итоге получаются искомые связи для парциальных фаддеевских компонент.

\section{2. ОСНОВНЫЕ ОПРЕДЕЛЕНИЯ И КЛЮЧЕВЫЕ ФОРМУЛЫ}

Будем использовать систему единиц, в которой заряд электрона $e$ и постоянная Планка $\hbar$ равны единице. В трехмерном координатном пространстве $\mathbb{R}^{3}$ фиксируем декартову систему координат $S$ с ортами $\hat{\mathbf{e}}_{1}, \hat{\mathbf{e}}_{2}$ и $\hat{\mathbf{e}}_{3}$ и начальной точкой $O$, совпадающей с центром масс исследуемой системы $\left\{p_{1}, p_{2}, p_{3}\right\}$ трех частиц $p_{1}, p_{2}$ и $p_{3}$ с массами $m_{1}, m_{2}, m_{3}$ и зарядами $z_{1}, z_{2}, z_{3}$. Пусть в этой системе $\mathbf{a}_{i j}-$ разность радиус-векторов $\mathbf{a}_{i}$ и $\mathbf{a}_{j}$ частиц $p_{i}$ и $p_{j}$, а $\mathbf{x}_{k}$ и $\mathbf{y}_{k}$ - приведенные векторы Якоби [9],

$$
\begin{aligned}
\mathbf{x}_{k} & \equiv \sqrt{2 \mu_{i j}}\left(\mathbf{a}_{j}-\mathbf{a}_{i}\right), & \mu_{i j} & \equiv \frac{m_{i} m_{j}}{m_{i}+m_{j}}, \\
\mathbf{y}_{k} & \equiv \sqrt{2 \mu_{k, i j}}\left(\frac{m_{i} \mathbf{a}_{i}+m_{j} \mathbf{a}_{j}}{m_{i}+m_{j}}-\mathbf{a}_{k}\right), & \mu_{k, i j} & \equiv \frac{m_{k}\left(m_{i}+m_{j}\right)}{m_{1}+m_{2}+m_{3}}
\end{aligned}
$$


где индексы $i, j, k$ образуют циклическую перестановку индексов $1,2,3$ : индекс $i$ переходит в $k, j-$ в $i$, а $k$ - в $j$. В шестимерном координатном пространстве $\mathbb{R}^{6}$ векторам $\mathbf{x}_{k}$ и $\mathbf{y}_{k}$ сопоставим вектор $\mathbf{r}_{k}=\left(\mathbf{x}_{k}, \mathbf{y}_{k}\right)$.

Выберем пару $\left\{p_{j}, p_{k}\right\}$ частиц $p_{j}$ и $p_{k}$. $\mathrm{B} \mathbb{R}^{6}$ под окрестностью точки парного удара частиц $p_{j}$ и $p_{k}$ подразумевается область $\mathcal{G} \equiv\left\{\mathbf{r}_{i}: x_{i} \ll 1, y_{i}>0\right\}$, в которой эти частицы близки друг к другу, но отделены от частицы $p_{i}$.

Пусть $\Psi^{\varepsilon}-$ регулярное $\left(\left|\Psi^{\varepsilon}(\mathbf{r})\right|<\infty \forall \mathbf{r}\right)$ решение уравнения Шредингера для системы $\left\{p_{1}, p_{2}, p_{3}\right\}$ в $\mathbb{R}^{6}$ :

$$
(H-E) \Psi^{\varepsilon}=0, \quad H=H_{0}+V, \quad V \equiv \sum_{k=1}^{3} V_{k},
$$

где $H_{0}$ - свободный гамильтониан, $E$ - полная энергия, $V_{k}-$ взаимодействие между частицами $p_{i}$ и $p_{j}$, а $\varepsilon$ - полный набор сохраняющихся квантовых чисел рассматриваемой трехчастичной системы.

В теории Фаддеева [9] решение $\Psi^{\varepsilon}$ рассматривается как сумма его трех фаддеевских компонент $\Psi_{1}^{\varepsilon}, \Psi_{2}^{\varepsilon}$ и $\Psi_{3}^{\varepsilon}$, подчиненных в $\mathbb{R}^{6}$ системе трех дифференциальных уравнений

$$
\left(H_{0}-E\right) \Psi_{k}^{\varepsilon}=-V_{k} \Psi^{\varepsilon}, \quad \Psi^{\varepsilon}=\Psi_{1}^{\varepsilon}+\Psi_{2}^{\varepsilon}+\Psi_{3}^{\varepsilon}, \quad k=1,2,3 .
$$

В случае центральных парных взаимодействий, например кулоновских

$$
V_{k}\left(x_{k}\left(a_{i j}\right)\right)=\frac{z_{i} z_{j}}{a_{i j}}, \quad V_{k}\left(x_{k}\right)=\frac{q_{k}}{x_{k}}, \quad q_{k} \equiv z_{i} z_{j} \sqrt{2 \mu_{i j}}, \quad k=1,2,3,
$$

или взаимодействий более общего вида

$$
V_{k}\left(x_{k}\right)=\frac{q_{k}}{x_{k}}+\bar{V}_{k}\left(x_{k}\right), \quad \bar{V}_{k}\left(x_{k}\right)=\sum_{n=0}^{\infty} x_{k}^{n} \bar{V}_{k n}, \quad \bar{V}_{k n}=\text { const }, \quad k=1,2,3,
$$

с гамильтонианом $H$ коммутируют сам $H$, квадрат оператора полного углового момента $\mathbf{l}$, его компонента $l_{3}$ и оператор $P_{r}$ инверсии $\mathbf{r}_{i} \rightarrow-\mathbf{r}_{i}$. Набор $\varepsilon=\{E, \ell, m, \sigma\}$ состоит из собственных чисел этих операторов.

Пусть некулоновские слагаемые $\bar{V}_{k}$ парных взаимодействий (5) являются аналитическими функциями. Полагаем, что

$$
x \equiv x_{i} \rightarrow 0, \quad y \equiv y_{i}>0, \quad u \equiv \cos \theta=\frac{(\mathbf{x} \cdot \mathbf{y})}{x y}, \quad k \neq i, \quad q \equiv q_{i},
$$

и используем координатное представление $\langle\mathbf{x}, \mathbf{y}| \equiv\left\langle\mathbf{x}_{i}, \mathbf{y}_{i}\right|$, в котором

$$
H_{0}=-\partial_{x}^{2}-\frac{2}{x} \partial_{x}+\frac{\mathbf{l}_{x}^{2}}{x^{2}}-\partial_{y}^{2}-\frac{2}{y} \partial_{y}+\frac{\mathbf{l}_{y}^{2}}{y^{2}}, \quad \mathbf{l}_{x} \equiv-i \mathbf{x} \times \nabla_{x}, \quad \mathbf{l}_{y} \equiv-i \mathbf{y} \times \nabla_{y} .
$$

В качестве собственных функций операторов $\mathrm{l}^{2}, l_{3}, P_{r}$ используем бисферические гармоники [16]

$$
\mathcal{Y}_{a b}^{\ell m}(\hat{x}, \hat{y}) \equiv \sum_{\alpha=-a}^{a} C_{a \alpha b \beta}^{\ell m} Y_{a \alpha}(\hat{y}) Y_{b \beta}(\hat{x}), \quad \mathbf{a}+\mathbf{b}=\mathbf{1}, \quad(-1)^{a+b}=\sigma
$$


а симметризованные $D$-функции Вигнера представим в виде [4]

$$
D_{m m^{\prime}}^{\ell \sigma}\left(\omega^{t}\right)=\left[\frac{2 \ell+1}{16 \pi^{2}\left(1+\delta_{m^{\prime} 0}\right)}\right]^{1 / 2}\left[D_{m m^{\prime}}^{\ell}\left(\omega^{t}\right)+\sigma(-1)^{\ell-m^{\prime}} D_{m,-m^{\prime}}^{\ell}\left(\omega^{t}\right)\right],
$$

где $C_{a \alpha b \beta}^{\ell m}-$ коэффициент Клебша-Гордана, $Y_{f e}-$ сферическая гармоника,

$$
Y_{f e}(\hat{a})=\frac{1}{\sqrt{2 \pi}} e^{-i e \varphi_{a}} \Theta_{f e}\left(\cos \theta_{a}\right), \quad \hat{a} \equiv\left(\theta_{a}, \varphi_{a}\right), \quad a=\hat{x}, \hat{y}
$$

$\omega^{t}=\left(\alpha^{t}, \beta^{t}, \gamma^{t}\right)$ - набор углов Эйлера, определяющих ориентацию выбранной “подвижной" системы координат $S^{t}$ относительно системы $S$.

Введем две "подвижные" системы координат $S^{t}=\left(\mathbf{e}_{1}^{t}, \mathbf{e}_{2}^{t}, \mathbf{e}_{3}^{t}\right), t=x, y$. Пусть начальные точки $O^{x}$ и $O^{y}$ систем $S^{x}$ и $S^{y}$ совпадают с начальной точкой $O$ фиксированной системы $S=\left(\mathbf{e}_{1}, \mathbf{e}_{2}, \mathbf{e}_{3}\right)$, а их реперы таковы, что

$$
\begin{array}{lll}
\left(\mathbf{y} \cdot \mathbf{e}_{1}^{x}\right)>0, & \left(\mathbf{y} \cdot \mathbf{e}_{2}^{x}\right)=0, & \left(\mathbf{x} \cdot \mathbf{e}_{3}^{x}\right)=x \\
\left(\mathbf{x} \cdot \mathbf{e}_{1}^{y}\right)<0, & \left(\mathbf{x} \cdot \mathbf{e}_{2}^{y}\right)=0, & \left(\mathbf{y} \cdot \mathbf{e}_{3}^{y}\right)=y .
\end{array}
$$

Тогда орты $\mathbf{e}_{2}^{x}$ и $\mathbf{e}_{2}^{y}$ направлены вдоль нормали $\mathbf{n}=\mathbf{x} \times \mathbf{y}$ к плоскости трех частиц, а система $S^{x}$ получается поворотом системы $S^{y}$ вокруг орта $\mathbf{e}_{2}^{y}$ на угол $\theta$ между векторами х и у. В системе $S$ ориентация этих векторов задана углами $\hat{x}=\left(\theta_{x}, \varphi_{x}\right)$ и $\hat{y}=\left(\theta_{y}, \varphi_{y}\right)$, а в системе $S^{t}-$ углами $\hat{x}^{t}$ и $\hat{y}^{t}$ :

$$
\begin{array}{lll}
\hat{x}^{t}=\left(\theta_{x}^{x}, \varphi_{x}^{x}\right)=(0,0), & \hat{y}^{t}=\left(\theta_{y}^{x}, \varphi_{y}^{x}\right)=(\theta, 0), & t=x, \\
\hat{x}^{t}=\left(\theta_{x}^{y}, \varphi_{x}^{y}\right)=(\theta, \pi), & \hat{y}^{t}=\left(\theta_{y}^{y}, \varphi_{y}^{y}\right)=(0,0), & t=y,
\end{array}
$$

поэтому переход $S \rightarrow S^{x}$ определяется углами Эйлера $\omega^{x}=\left(\varphi_{x}, \theta_{x}, \gamma^{x}\right)$, а переход $S \rightarrow S^{y}$ - углами Эйлера $\omega^{y}=\left(\varphi_{y}, \theta_{y}, \gamma^{y}\right)$, где углы $\gamma^{t}$ таковы, что

$$
\cos \gamma^{t}=\operatorname{ctg} \theta \cos \theta_{t}-\operatorname{cosec} \theta \cos \theta_{t^{\prime}}, \quad t, t^{\prime}=x, y \quad t^{\prime} \neq t
$$

Далее будут рассматриваться два случая $t=x$ и $t=y$. В случае $t=x$ "подвижной" является система $S^{x}$, а в случае $t=y$ - система $S^{y}$.

Выведем ключевые формулы дифференцирования.

Векторы Якоби (1) кинематически связаны [17]:

$$
\left(\begin{array}{l}
\mathbf{x}_{k} \\
\mathbf{y}_{k}
\end{array}\right)=-\left(\begin{array}{cc}
c_{k i} & s_{k i} \\
-s_{k i} & c_{k i}
\end{array}\right)\left(\begin{array}{c}
\mathbf{x}_{i} \\
\mathbf{y}_{i}
\end{array}\right), \quad\left(\begin{array}{c}
c_{k i} \\
s_{k i}
\end{array}\right) \equiv\left(\begin{array}{c}
\cos \gamma_{k i} \\
\sin \gamma_{k i}
\end{array}\right), \quad \gamma_{k i} \in\left[-\frac{\pi}{2}, \frac{\pi}{2}\right],
$$

где $\gamma_{k i}$ - кинематический угол, зависящий только от отношений масс частиц. В силу такой связи $x_{k}, y_{k}-$ функции аргументов $x, y, u$ :

$$
\begin{aligned}
& \left\langle\mathbf{x}, \mathbf{y} \mid x_{k}\right\rangle=x_{k}(x, y, u)=\left[\left(c_{k i} x\right)^{2}+\left(s_{k i} y\right)^{2}+2 c_{k i} s_{k i} u x y\right]^{1 / 2}, \\
& \left\langle\mathbf{x}, \mathbf{y} \mid y_{k}\right\rangle=y_{k}(x, y, u)=\left[\left(s_{k i} x\right)^{2}+\left(c_{k i} y\right)^{2}-2 c_{k i} s_{k i} u x y\right]^{1 / 2},
\end{aligned}
$$


поэтому

$$
\begin{gathered}
\left\langle\mathbf{x}, \mathbf{y} \mid x_{k}\right\rangle=x_{k}(x, y, u)=\frac{\left|s_{k i}\right| y}{g\left(v_{x}\right)}, \quad\left\langle\mathbf{x}, \mathbf{y} \mid y_{k}\right\rangle=y_{k}(x, y, u)=\frac{c_{k i} y}{g\left(v_{y}\right)}, \\
g(v) \equiv\left(1-2 u v+v^{2}\right)^{-1 / 2}, \quad v_{x} \equiv-\frac{c_{k i} x}{s_{k i} y}, \quad v_{y} \equiv \frac{s_{k i} x}{c_{k i} y} .
\end{gathered}
$$

Следовательно, функция $1 / x_{k}(x, y, u)$ пропорциональна производящей функции $g\left(v_{x}\right)$ для полиномов Лежандра $P_{n}(u)$ (см. [16]), поэтому

$$
\frac{1}{x_{k}}=\frac{1}{\left|s_{k i}\right| y} \sum_{n=0}^{\infty}\left(-\frac{c_{k i} x}{s_{k i} y}\right)^{n} P_{n}(u), \quad k \neq i
$$

Из соотношений (11), (12) вытекает формула дифференцирования

$$
\left.\partial_{x}^{n} x_{k}\right|_{x=0}=c_{k i} \frac{s_{k i}}{\left|s_{k i}\right|} \frac{n !}{2 n-1}\left(-\frac{c_{k i}}{s_{k i} y}\right)^{n-1}\left[P_{n}(u)-P_{n-2}(u)\right], \quad n=0,1, \ldots
$$

Аналогичным образом доказывается формула дифференцирования

$$
\left.\partial_{x}^{n} y_{k}\right|_{x=0}=s_{k i} \frac{n !}{2 n-1}\left(\frac{s_{k i}}{c_{k i} y}\right)^{n-1}\left[P_{n}(u)-P_{n-2}(u)\right], \quad n=0,1, \ldots
$$

Теперь приведем и поясним разложения, доказанные в работе [8] и являющиеся ключевыми для построений, выполненных в разделе 3 .

Гладкая часть $\bar{V}_{k}$ парного взаимодействия (5) является рядом

$$
\bar{V}_{k}\left(x_{k}(x, y, u)\right)=\sum_{p=0}^{\infty} x^{p} \sum_{s=0}^{p} \bar{V}_{k}^{p s}\left(\left|s_{k i}\right| y\right) P_{s}(u), \quad(-1)^{p+s}=1 .
$$

С учетом представлений (5), (12), (15) полное взаимодействие $V=V_{1}+V_{2}+V_{3}$ разлагается в двойной ряд

$$
\begin{gathered}
V(x, y, u)=\frac{q}{x}+V^{00}(y)+\sum_{p=1}^{\infty} x^{p} \sum_{s=0}^{p} V^{p s}(y) P_{s}(u), \\
V^{p s}(y) \equiv \bar{V}_{i p} \delta_{s 0}+\delta_{p s} \sum_{k \neq i} \frac{q_{k}}{\left|s_{k i}\right| y}\left(-\frac{c_{k i}}{s_{k i} y}\right)^{p}+\sum_{k \neq i} \bar{V}_{k}^{p s}(y),
\end{gathered}
$$

где отличны от нуля только константы $V^{p 0}=\bar{V}_{i p}$ с нечетным $p$ и функции $V^{p s}(y)$ с четной суммой $p+s$. При $p \leqslant 2$ их можно найти по формулам

$$
\begin{aligned}
& V^{00}(y)=V_{i 0}+\sum_{k \neq i}\left[\frac{q_{k}}{\left|s_{k i}\right| y}+\bar{V}_{k}\left(\left|s_{k i}\right| y\right)\right], \quad V^{10}(y)=V_{i 1}, \\
& V^{11}(y)=\sum_{k \neq i} c_{k i}\left(\frac{s_{k i}}{\left|s_{k i}\right|}\right)\left[\bar{V}_{k}^{\prime}\left(\left|s_{k i}\right| y\right)-\frac{q_{k}}{\left(s_{k i} y\right)^{2}}\right], \\
& V^{20}(y)=V_{i 2}+\frac{1}{6} \sum_{k \neq i} c_{k i}^{2}\left[\bar{V}_{k}^{\prime \prime}\left(\left|s_{k i}\right| y\right)+\frac{2 \bar{V}_{k}^{\prime}\left(\left|s_{k i}\right| y\right)}{\left|s_{k i}\right| y}\right], \\
& V^{22}(y)=\sum_{k \neq i}\left\{\frac{q_{k} c_{k i}^{2}}{\left(\left|s_{k i}\right| y\right)^{3}}+\frac{1}{3} c_{k i}^{2}\left[\frac{\bar{V}_{k}^{\prime \prime}\left(\left|s_{k i}\right| y\right)-\bar{V}_{k}^{\prime}\left(\left|s_{k i}\right| y\right)}{\left|s_{k i}\right| y}\right]\right\},
\end{aligned}
$$


где $\bar{V}_{k}^{\prime}$ и $\bar{V}_{k}^{\prime \prime}-$ первая и вторая производные функции $\bar{V}_{k}\left(x_{k}\right)$ по аргументу $x_{k}$ в точке $x_{k}=\left|s_{k i}\right| y$, т.е. при $x=0$.

Разложение решения $\Psi^{\varepsilon}$ по бисферическому базису (7) можно представить конечной суммой

$$
\Psi^{\varepsilon}(\mathbf{x}, \mathbf{y})=\sum_{a b} \Psi_{a b}^{\varepsilon}(x, y) \mathcal{Y}_{a b}^{\ell m}(\hat{x}, \hat{y}), \quad \mathbf{a}+\mathbf{b}=\mathbf{l}, \quad(-1)^{a+b}=\sigma
$$

где ее бисферические компоненты $\Psi_{a b}^{\varepsilon}$ являются рядами

$$
\Psi_{a b}^{\varepsilon}(x, y)=\sum_{n=b \geqslant \mu(\sigma)}^{\infty} x^{n} \Psi_{a b}^{n \varepsilon}(y), \quad \mu(\sigma) \equiv \frac{1}{2}\left[1-\sigma(-1)^{\ell}\right],
$$

а компоненты $\Psi_{a b}^{n \varepsilon}(y)$ этих рядов подчинены цепочкам рекуррентных по индексу $n \geqslant b$ алгебраических уравнений и однозначно выражаются через неопределенные функции $\Psi_{a b}^{n \varepsilon}(y), b=n$, и известные коэффициенты $q_{k}, \bar{V}_{i p}$ и функции $\bar{V}^{p s}(y)$, содержащиеся в разложении (16) полного взаимодействия.

Разложение решения $\Psi^{\varepsilon}$ по $D^{\sigma}$-базису является конечной суммой

$$
\Psi^{\varepsilon}(\mathbf{x}, \mathbf{y})=\sum_{m^{\prime}=\mu(\sigma)}^{\ell} \Psi_{m^{\prime}}^{\varepsilon t}(x, y, \theta) D_{m m^{\prime}}^{\ell \sigma *}\left(\omega^{t}\right), \quad t=x, y .
$$

Все ее $D^{\sigma}$-компоненты $\Psi_{m^{\prime}}^{\varepsilon t}$ в случае $t=x$ являются рядами

$$
\Psi_{m^{\prime}}^{\varepsilon x}(x, y, \theta)=\sum_{n=m^{\prime}}^{\infty} x^{n} \sum_{a=m^{\prime}}^{\ell+n-\mu(\sigma)} \Psi_{a m^{\prime}}^{n \varepsilon x}(y) \Theta_{a m^{\prime}}(u)
$$

а в случае $t=y-$ рядами

$$
\Psi_{m^{\prime}}^{\varepsilon y}(x, y, \theta)=\sum_{n=\mu(\sigma)}^{\infty} x^{n} \sum_{b=m^{\prime}}^{n} \Psi_{b m^{\prime}}^{n \varepsilon y}(y) \Theta_{b m^{\prime}}(u)
$$

В обоих случаях $\Theta$-компоненты $\Psi_{a m^{\prime}}^{n \varepsilon t}(y)$ подчиняются цепочкам рекуррентных по индексу $n \geqslant b$ алгебраических уравнений и однозначно выражаются через соответствующие $(t=x$ или $t=y)$ произвольные функции $f_{m^{\prime}}^{n+2, t}(y)$, известные коэффициенты $q_{k}, \bar{V}_{i p}$ и компоненты $\bar{V}^{p s}(y)$ разложения (16).

\section{3. СЛУЧАЙ ЦЕНТРАЛЬНЫХ ПАРНЫХ ВЗАИМОДЕЙСТВИЙ}

Исследуем строение фаддеевской компоненты $\Psi_{i}^{\varepsilon}$ в бисферическом базисе.

Пусть решение $\Psi^{\varepsilon}$ уравнения Шредингера представлено рядом (17) с известными компонентами $\Psi_{a b}^{n \varepsilon}(y)(18)$. Докажем, что такое представление порождает аналогичные представления фаддеевской компоненты

$$
\Psi_{i}^{\varepsilon}(\mathbf{x}, \mathbf{y})=\sum_{a b} \Psi_{i a b}^{\varepsilon}(x, y) \mathcal{Y}_{a b}^{\ell m}(\hat{x}, \hat{y}), \quad \mathbf{a}+\mathbf{b}=\mathbf{1}, \quad(-1)^{a+b}=\sigma
$$


и ее бисферических компонент

$$
\Psi_{i a b}^{\varepsilon}(x, y) \equiv\left\langle\mathcal{Y}_{a b}^{\ell m}(\hat{x}, \hat{y}) \mid \Psi_{i}^{\varepsilon}(\mathbf{x}, \mathbf{y})\right\rangle=\sum_{n=b \geqslant \mu(\sigma)}^{\infty} x^{n} \Psi_{i a b}^{n \varepsilon}(y)
$$

Для этого исследуем только одно уравнение системы Фаддеева (3), а именно уравнение, содержащее в левой части компоненту $\Psi_{i}^{\varepsilon}$. В этом уравнении представим операторы $V_{i}, H_{0}$ и решение $\Psi^{\varepsilon}$ уравнения Шредингера соответствующими суммами $(5),(6)$ и $(17),(18)$. Правая часть получившегося уравнения является рядом по степеням $x$ и базисным функциям $\mathcal{Y}_{a b}^{\ell m}$. Поэтому искомое регулярное решение $\Psi_{i}^{\varepsilon}$ может быть рядом только того же типа, но пока без дополнительных ограничений на индексы $a, b$ и $n$ :

$$
\Psi_{i}^{\varepsilon}(\mathbf{x}, \mathbf{y})=\sum_{n=0}^{\infty} x^{n} \sum_{a b} \Psi_{i a b}^{n \varepsilon}(y) \mathcal{Y}_{a b}^{\ell m}(\hat{x}, \hat{y}) .
$$

Заменим компоненту $\Psi_{i}^{\varepsilon}$ таким рядом. Спроецируем полученное уравнение на функции $\mathcal{Y}_{a b}^{\ell m}(\hat{x}, \hat{y})$ и выведем для каждой пары $\{a, b\}$ алгебраическую и рекуррентную по индексу $n$ цепочку уравнений

$$
\begin{gathered}
{[b(b+1)-(n+2)(n+3)] \Psi_{i a b}^{n+2, \varepsilon}(y)=-\left[h_{a}(y)-V^{00}(y)\right] \Psi_{i a b}^{n \varepsilon}(y)-} \\
-q \Psi_{a b}^{n+1, \varepsilon}(y)-\sum_{p=0}^{n} V_{i p} \Psi_{a b}^{n-p, \varepsilon}(y), \quad n=-2,-1, \ldots
\end{gathered}
$$

где $h_{a}$ - диагональный матричный элемент оператора

$$
h(\mathbf{y}) \equiv-\partial_{y}^{2}-\frac{2}{y} \partial_{y}+\frac{\mathbf{l}_{y}^{2}}{y^{2}}+V^{00}(y)-E
$$

в бисферическом базисе (7)

$$
\begin{gathered}
\left\langle\mathcal{Y}_{a b}^{\ell m}|h(\mathbf{y})| \mathcal{Y}_{a^{\prime} b^{\prime}}^{\ell m}\right\rangle=\delta_{a a^{\prime}} \delta_{b b^{\prime}} h_{a}(y), \\
h_{a}(y) \equiv-\partial_{y}^{2}-\frac{2}{y} \partial_{y}+\frac{a(a+1)}{y^{2}}+V^{00}(y)-E
\end{gathered}
$$

Пусть в этой цепочке $n<b$ или $b<\mu(\sigma)$, тогда $\Psi_{a b}^{n \varepsilon}(y) \equiv 0$, поэтому $\Psi_{i a b}^{n \varepsilon}(y) \equiv 0$. Далее полагаем $n \geqslant b \geqslant \mu(\sigma)$. При таких ограничениях пробный ряд (24) становится рядом (22), а исследуемая цепочка однозначно разрешима: при данных $a$ и $b$ все искомые функции $\Psi_{i a b}^{n \varepsilon}$ при $n>b$ выражаются через неопределенную функцию $\Psi_{i a b}^{n \varepsilon}(y)$ с $n=b$ и известные функции $\Psi_{a b}^{n \varepsilon}$. Следовательно, разложения (22) и (23) существуют, что и требовалось доказать.

Теперь построим явные асимптотики рядов $(23)$ при $b \leqslant 2$ как подсуммы их трех слагаемых наиболее медленно убывающих при $x \rightarrow 0$, а затем получим связи при $x=0$ для производных $\partial_{x}^{n} \Psi_{i a b}^{\varepsilon}$. Сначала в уравнениях (25) заменим функции $\Psi_{a b}^{n \varepsilon}(y)$ при $n \leqslant 3$ их явными выражениями, найденными в работе [8]. Решим полученные уравнения и выразим функции $\Psi_{i a b}^{n \varepsilon}(y)$ как комбинации неопределенных функций 
$\Psi_{a b}^{n \varepsilon}$ и $\Psi_{i a b}^{n \varepsilon}$ с $n=b$, равных производным $(n !)^{-1} \partial_{x}^{n} \Psi_{a b}^{\varepsilon}(x, y)$ и $(n !)^{-1} \partial_{x}^{n} \Psi_{i a b}^{\varepsilon}(x, y)$ при $x=0$ и $n=b$. Используя такие комбинации, получаем следующие асимптотические формулы для компонент рядов (23):

$$
\begin{aligned}
\Psi_{i \ell 0}^{\varepsilon}(x, y)=\{1 & \left.+\frac{x^{2}}{6}\left(h_{\ell}-V^{00}\right)\left[1+\frac{x^{2}}{20}\left(h_{\ell}-V^{00}\right)\right]\right\} \Psi_{i \ell 0}^{\varepsilon}(0, y)+ \\
& +\frac{x}{2}\left\{q+\frac{x}{6}\left(2 V_{i 0}+q^{2}\right)+\frac{x^{2}}{72}\left[q^{3}+2 q\left(4 h_{\ell}+3 V_{i 0}-3 V^{00}\right)+12 V_{i 1}\right]+\right. \\
& +\frac{x^{3}}{1440}\left[q^{4}+4 q^{2}\left(5 h_{\ell}+3 V_{i 0}-3 V^{00}\right)+\right. \\
& \left.\left.+84 q V_{i 1}+24\left(6 V_{i 2}+V_{i 0}\left(2 h_{\ell}-V^{00}\right)\right)\right]\right\} \Psi_{\ell 0}^{\varepsilon}(0, y)+O\left(x^{5}\right) \\
\Psi_{i a 1}^{\varepsilon}(x, y)=x[ & \left.+\frac{x^{2}}{10}\left(h_{a}-V^{00}\right)\right]\left.\partial_{x} \Psi_{i a 1}^{\varepsilon}(x, y)\right|_{x=0}+ \\
& +\frac{x^{2}}{4}\left\{q+\frac{x}{10}\left(4 V_{i 0}+q^{2}\right)+\right. \\
& \left.+\frac{x^{2}}{180}\left[q^{3}+2 q\left(7 h_{a}+5 V_{i 0}-5 V^{00}\right)+40 V_{i 1}\right]\right\}\left.\partial_{x} \Psi_{a 1}^{\varepsilon}(x, y)\right|_{x=0}- \\
& -\frac{x^{4}}{180 \sqrt{3}} q V^{11} C_{\ell 010}^{a 0} \Psi_{\ell 0}^{\varepsilon}(0, y)+O\left(x^{5}\right)
\end{aligned}
$$

где $a=|\ell \pm(1-\mu(\sigma))|$,

$$
\begin{aligned}
\Psi_{i a 2}^{\varepsilon}(x, y)=\frac{x^{2}}{2} & {\left.\left[1+\frac{x^{2}}{14}\left(h_{a}-V^{00}\right)\right] \partial_{x}^{2} \Psi_{i 2 a}^{\varepsilon}(x, y)\right|_{x=0}+} \\
& +\left.\frac{x^{3}}{12}\left[q+\frac{x}{14}\left(6 V_{i 0}+q^{2}\right)\right] \partial_{x}^{2} \Psi_{2 a}^{\varepsilon}(x, y)\right|_{x=0}+O\left(x^{5}\right)
\end{aligned}
$$

где $a=|\ell+\mu(\sigma)|, \mid \ell \pm\left(2-\mu(\sigma) \mid\right.$. Вычислив производные $\partial_{x}^{n}, n \leqslant 4$, этих асимптотик, получаем серию связей при $x=0$ и $y>0$ для компонент $\Psi_{i a b}^{\varepsilon}$ и $\Psi_{a b}^{\varepsilon}$. В этой серии при любом $q$ имеются связи

$$
\partial_{x}^{b}\left[2 \partial_{x} \Psi_{i a b}^{\varepsilon}(x, y)-q \Psi_{a b}^{\varepsilon}(x, y)\right]=0, \quad b \geqslant n=0,1,2
$$

Из этих связей в случае $q \neq 0$ следуют равенства

$$
\partial_{x}^{b} \Psi_{a b}^{\varepsilon}(x, y)=\frac{2}{q} \partial_{x}^{b+1} \Psi_{a b}^{\varepsilon}(x, y), \quad b=0,1,2, \quad x=0, \quad y>0
$$

Используя эти равенства, исключим производные $\partial_{x}^{b} \Psi_{a b}^{\varepsilon}$ из других связей исследуемой серии и в результате выведем связи, но уже только для фаддеевских компо- 
нент $\Psi_{i a b}^{\varepsilon}$ :

$$
\begin{aligned}
& {\left[3 q \partial_{x}^{2}-\left(2 V_{i 0}+q^{2}\right) \partial_{x}-q\left(h_{\ell}-V^{00}\right)\right] \Psi_{i \ell 0}^{\varepsilon}(x, y) }=0, \\
& \partial_{x}\left\{12 q \partial_{x}^{2}-\left[q^{3}+2 q\left(4 h_{\ell}+3 V_{i 0}-3 V^{00}\right)+12 V_{i 1}\right]\right\} \Psi_{i \ell 0}^{\varepsilon}(x, y)=0, \\
&\left\{60 q \partial_{x}^{4}-\left[q^{4}+4 q^{2}\left(5 h_{\ell}+3 V_{i 0}-3 V^{00}\right)+84 q V_{i 1}+\right.\right. \\
&\left.\left.+24\left(6 V_{i 2}+V_{i 0}\left(2 h_{\ell}-V^{00}\right)\right)\right] \partial_{x}-12 q\left(h_{\ell}-V^{00}\right)^{2}\right\} \Psi_{i \ell 0}^{\varepsilon}(x, y)=0, \\
& \partial_{x}\left[10 q \partial_{x}^{2}-3\left(4 V_{i 0}+q^{2}\right) \partial_{x}-3\left(h_{a}-V^{00}\right)\right] \Psi_{i a 1}^{\varepsilon y}(x, y)=0, \\
& \sqrt{3} \partial_{x}^{2}\left\{15 q \partial_{x}^{2}-\left[q^{2}+2 q\left(7 h_{a}+5 V_{i 0}-5 V^{00}\right)+40 V_{i 1}\right]\right\} \Psi_{i a 1}^{\varepsilon y}(x, y)==4 q V^{11} C_{\ell 010}^{a 0} \partial_{x} \Psi_{i \ell 0}^{\varepsilon y}(x, y) \\
&=0, \\
& \partial_{x}^{2}\left[7 q \partial_{x}^{2}-2 \partial_{x}-6\left(h_{a}-V^{00}\right)\right] \Psi_{i a 2}^{\varepsilon}(x, y)=0 .
\end{aligned}
$$

Если $q=0$ и $x=0$, то все связи (30) и (31) становятся тривиальными: $\partial_{x}^{b+1} \Psi_{i a b}^{\varepsilon}=0$, $b=0,1,2$, между компонентами $\Psi_{i a b}^{\varepsilon}$ и $\Psi_{a b}^{\varepsilon}$ имеются связи

$$
\begin{aligned}
\left(3 \partial_{x}^{2}+V^{00}-h_{\ell}\right) \Psi_{i \ell 0}^{\varepsilon}(x, y) & =V_{i 0} \Psi_{\ell 0}^{\varepsilon}(x, y), \\
\partial_{x}\left[5 \partial_{x}^{2}-3\left(h_{a}-V^{00}\right)\right] \Psi_{i a 1}^{\varepsilon}(x, y) & =3 V_{i 0} \partial_{x} \Psi_{a 1}^{\varepsilon}(x, y), \\
\partial_{x}^{2}\left[7 \partial_{x}^{2}-6\left(h_{a}-V^{00}\right)\right] \Psi_{i a 2}^{\varepsilon}(x, y) & =6 \partial_{x}^{2} \Psi_{a 2}^{\varepsilon}(x, y),
\end{aligned}
$$

а компоненты $\Psi_{i a b}^{\varepsilon}, b=0,1$, удовлетворяют следующим связям:

$$
\begin{aligned}
& {\left[2 V_{i 0} \partial_{x}^{3}-V_{i 1}\left(3 \partial_{x}^{2}+V^{00}-h_{\ell}\right)\right] \Psi_{i \ell 0}^{\varepsilon}(x, y) }=0 \\
&\left\{5 V_{i 0} \partial_{x}^{4}-3\left[6 V_{i 2}+V_{i 0}\left(2 h_{\ell}-V^{00}\right)\right] \partial_{x}^{2}+\right. \\
&\left.+\left(6 V_{i 2}+V_{i 0} h_{\ell}\right)\left(h_{\ell}-V^{00}\right)\right\} \Psi_{i \ell 0}^{\varepsilon}(x, y)=0 \\
& \partial_{x}\left[9 \partial_{x}^{3}-20 \partial_{x}^{2}+12\left(h_{a}-V^{00}\right)\right] \Psi_{i a 1}^{\varepsilon}(x, y)=0 .
\end{aligned}
$$

Как известно [9], шестимерные уравнения Фаддеева (3) подстановкой представления (22) сводятся к системе двумерных интегродифференциальных уравнений для совокупности $\left\{\Psi_{j a b}^{\varepsilon}\right\}$ всех фаддеевских бисферических компонент $\Psi_{j a b}^{\varepsilon}$. Ядра интегральных операторов таких двумерных уравнений Фаддеева являются довольно сложными функциями. Детальное исследование этих ядер выполнено в работах [18], [19]. Сравнительный анализ дискретных сплайн-аналогов двумерных уравнений Фаддеева дан в обзоре [12]. Во все такие аналоги несложно включить полученные связи (30)-(33) и улучшить поточечную сходимость вычисляемого решения к точному решению $\left\{\Psi_{j a b}^{\varepsilon}\right\}$ вблизи точки парного удара. Пример такого включения дан в работе [13].

Теперь исследуем строение фаддеевской компоненты $\Psi_{i}^{\varepsilon}$ в $D^{\sigma}$-базисе.

Пусть решение $\Psi^{\varepsilon}$ уравнения Шредингера представлено рядом (19) с $D^{\sigma}$-компонентами (20) или рядом (19) с $D^{\sigma}$-компонентами (21), а все компоненты этих рядов $\Psi_{i m^{\prime}}^{n \varepsilon t}(y)$, где $t=x$ или $t=y$, известны. Используя те же приемы, что и при доказательстве формул (22), нетрудно показать, что обоим представлениям (при $t=x$ и $t=y)$ функции $\Psi^{\varepsilon}$ отвечают аналогичные по строению разложения фаддеевской 
компоненты $\Psi_{i}^{\varepsilon}$ и разложения ее $D^{\sigma}$-компонент $\Psi_{i m^{\prime}}^{\varepsilon t}:$ при $t=x$

$$
\begin{aligned}
\Psi_{i}^{\varepsilon}(\mathbf{x}, \mathbf{y}) & =\sum_{m^{\prime}=\mu(\sigma)}^{\ell} D_{m m^{\prime}}^{\ell \sigma *}\left(\omega^{x}\right) \Psi_{i m^{\prime}}^{\varepsilon x}(x, y, \theta), \\
\Psi_{i m^{\prime}}^{\varepsilon x}(x, y, \theta) & =\sum_{n=\mu(\sigma)}^{\infty} x^{n} \sum_{a=m^{\prime}}^{\ell+n-\mu(\sigma)} \Psi_{i a m^{\prime}}^{n \varepsilon x}(y) \Theta_{a m^{\prime}}(u),
\end{aligned}
$$

при $t=y$

$$
\begin{aligned}
\Psi_{i}^{\varepsilon}(\mathbf{x}, \mathbf{y}) & =\sum_{m^{\prime}=\mu(\sigma)}^{\ell} D_{m m^{\prime}}^{\ell \sigma *}\left(\omega^{y}\right) \Psi_{i m^{\prime}}^{\varepsilon y}(x, y, \theta), \\
\Psi_{i m^{\prime}}^{\varepsilon y}(x, y, \theta) & =\sum_{n=\mu(\sigma)}^{\infty} x^{n} \sum_{b=m^{\prime}}^{n} \Psi_{i b m^{\prime}}^{n \varepsilon y}(y) \Theta_{b m^{\prime}}(u) .
\end{aligned}
$$

Вывод уравнений для неизвестных $\Theta$-компонент $\Psi_{i a m^{\prime}}^{n \varepsilon x}$ и $\Psi_{i b m^{\prime}}^{n \varepsilon y}$ из уравнения (3), содержащего в левой части компоненту $\Psi_{i}^{\varepsilon}$, несложен. Сначала $V_{i}, H_{0}$ и $\Psi^{\varepsilon}$ заменяются соответствующими суммами (5), (6) и (19), (20) или (19), (21), а $\Psi_{i}^{\varepsilon}$ - искомым рядом (34) или (36). Затем полученные уравнения проецируются сначала на функции $D_{m m^{\prime}}^{\ell \sigma *}\left(\omega^{t}\right)$ с $t=x$ или $t=y$, а затем на соответствующие базисные функции $\Theta_{a m^{\prime}}(u)$ или $\Theta_{b m^{\prime}}(u)$. Все правила для такого последовательного проецирования подробно описаны в работе [8].

В итоге для искомых $\Theta$-компонент $\Psi_{i a m^{\prime}}^{n \varepsilon t}(y), t=x, y$, получаются рекуррентные по индексу $n$ цепочки алгебраических уравнений. В случае $t=x$ при любых $m^{\prime}$ и $n>\mu(\sigma)$ уравнения цепочки для неизвестных $\Psi_{i a m^{\prime \prime}}^{n \varepsilon x}(y)$ зацепляются по индексу $m^{\prime \prime}=m^{\prime}, m^{\prime} \pm 1$; поэтому цепочка неудобна для анализа и далее не рассматривается. В случае $t=y$ для каждого $m^{\prime}=\mu(\sigma), \mu(\sigma)+1, \ldots, \ell$, получается простая рекуррентная $(n=-2,-1, \ldots)$ цепочка

$$
\begin{aligned}
& {[b(b+1)-(n+2)(n+3)] \Psi_{i b m^{\prime}}^{n+2, \varepsilon y}(y)=-q \Psi_{b m^{\prime}}^{n+1, \varepsilon y}(y)-\sum_{p=0}^{n} V_{i p} \Psi_{b^{\prime} m^{\prime}}^{n-p, \varepsilon y}(y)+} \\
& +y^{-2} \sum_{m^{\prime \prime}=m^{\prime} \pm 1} \gamma_{m^{\prime} m^{\prime \prime}}^{\ell \sigma} q_{m^{\prime} m^{\prime \prime}}^{b} \Psi_{i b m^{\prime \prime}}^{n \varepsilon y}(y)-\left[h_{b m^{\prime}}^{y}(y)-V^{00}(y)\right] \Psi_{i b m^{\prime}}^{n \varepsilon y}(y),
\end{aligned}
$$

где $\Psi_{i b m^{\prime}}^{n \varepsilon x} \equiv 0$ при $n<b$ и при $b<m^{\prime}, h_{b m^{\prime}}^{y}(y)$ - дифференциальный оператор,

$$
h_{b m^{\prime}}^{y}(y) \equiv-\frac{1}{y^{2}} \partial_{y}\left(y^{2} \partial_{y}\right)+\frac{1}{y^{2}}\left[\ell(\ell+1)+b(b+1)-2 m^{\prime 2}\right]+V^{00}(y)-E,
$$

а коэффициенты $\gamma_{m^{\prime} m^{\prime \prime}}^{\ell \sigma}$ и $q_{m^{\prime} m^{\prime \prime}}^{b}$ вычисляются по формулам

$$
\begin{aligned}
\gamma_{m^{\prime}, m^{\prime}+1}^{\ell \sigma} & \equiv\left\{\left[1+\delta_{m^{\prime} 0} \sigma(-1)^{\ell}\right]\left[\ell(\ell+1)-m^{\prime}\left(m^{\prime}+1\right)\right]\right\}^{1 / 2}, \\
\gamma_{m^{\prime}, m^{\prime}-1}^{\ell \sigma} & \equiv\left(1-\delta_{m^{\prime} 0}\right)\left\{\left[1+\delta_{m^{\prime} 1} \sigma(-1)^{\ell}\right]\left[\ell(\ell+1)-m^{\prime}\left(m^{\prime}-1\right)\right]\right\}^{1 / 2}, \\
q_{m^{\prime} m^{\prime \prime}}^{b} & \equiv\left[b(b+1)-m^{\prime} m^{\prime \prime}\right]^{1 / 2}, \quad m^{\prime \prime}=m^{\prime} \pm 1 .
\end{aligned}
$$


Решения $\Psi_{i b m^{\prime}}^{n+2, \varepsilon y}$ уравнений $(38)$ удобно искать в порядке возрастания $(n=$ $-2,-1, \ldots)$ индекса $n$ и убывания $(b=n, n-1, \ldots)$ индекса $b$ при каждом $n$. Уравнение (38) при $b=n+2$ имеет вид $0 \cdot \Psi_{i b m^{\prime}}^{n+2, \varepsilon y}=0$, поэтому $\Psi_{i m^{\prime}}^{n+2, \varepsilon y}(y)=g_{i m^{\prime}}^{n+2, y}(y)$, где $g_{i m^{\prime}}^{n+2, y}(y)$ - некоторая нетривиальная функция. Исключение составляет случай $\sigma=(-1)^{\ell+1}, m^{\prime}=0$, когда согласно $(37) \Psi_{i m^{\prime}}^{n \varepsilon y}=0$ при всех $n$. Поэтому в этом случае полагаем $g_{i 0}^{n y}(y) \equiv 0$ при всех $n$.

Теперь построим явные асимптотики рядов (37) при $m^{\prime} \leqslant 2$ в виде подсумм их трех наиболее медленно убывающих при $x \rightarrow 0$ слагаемых и выведем связи для $\Theta$-компонент $\Psi_{i b m^{\prime}}^{\varepsilon y}$. Сначала в уравнениях $(38)$ при $m^{\prime} \leqslant 2$ заменим функции $\Psi_{b m^{\prime}}^{n \varepsilon}(y)$ при $n \leqslant 3$ их явными выражениями, найденными в работе [8]. Затем решим полученные уравнения и выразим решения $\Psi_{i b m^{\prime}}^{n \varepsilon y}(y)$ через неопределенные функции $f_{m^{\prime}}^{n y} \equiv \Psi_{b m^{\prime}}^{n \varepsilon y}$ и $g_{i m^{\prime}}^{n y} \equiv \Psi_{i b m^{\prime}}^{n \varepsilon y}$, равные производным $(n !)^{-1} \partial_{x}^{n} \Psi_{m^{\prime}}^{\varepsilon y}(x, y)$ и $(n !)^{-1} \partial_{x}^{n} \Psi_{i m^{\prime}}^{\varepsilon y}(x, y)$ при $x=0$ и $n=b$. Используя полученные выражения, представим асимптотики рядов $(37)$ при $m^{\prime} \leqslant 2$, т.е. асимптотики $D^{\sigma}$-компонент $\Psi_{i m^{\prime}}^{\varepsilon y}$, $m^{\prime} \leqslant 2$, в обоих случаях $\sigma= \pm(-1)^{\ell}$ в виде

$$
\begin{aligned}
& \Psi_{i 0}^{\varepsilon y}(x, y, \theta)=\{ g_{i 0}^{0 y}+\frac{q x}{2} f_{0}^{0 y}+\frac{x^{2}}{12}\left[\left(2 V_{i 0}+q^{2}\right) f_{0}^{0 y}+2\left(h_{00}^{y}-V^{00}\right) g_{i 0}^{0 y}\right]+ \\
&\left.+\frac{x^{3}}{144}\left[q^{3}+2 q\left(4 h_{00}^{y}+3 V_{i 0}-3 V^{00}\right)+12 V_{i 1}\right] f_{0}^{0 y}\right\} \Theta_{00}(u)+ \\
&+x\left[g_{i 0}^{1 y}+\frac{q x}{4} f_{0}^{1 y}+\frac{x^{2}}{40}\left(4 V_{i 0}+q^{2}\right) f_{0}^{1 y}\right] \Theta_{10}(u)+ \\
&+\frac{x^{3}}{10 y^{2}}\left\{y^{2}\left[h_{10}^{y}-V^{00}\right] g_{i 0}^{1 y}-\left[2 \ell(\ell+1)\left(1+\sigma(-1)^{\ell}\right)\right]^{1 / 2} g_{i 1}^{1 y}\right\} \Theta_{10}(u)+ \\
&+x^{2}\left[g_{i 1}^{2 y}+\frac{q x}{6} f_{0}^{2 y}\right] \Theta_{20}(u)+x^{3} g_{i 0}^{3 y} \Theta_{30}(u)+O\left(x^{4}\right), \\
& \Psi_{i 1}^{\varepsilon y}(x, y, \theta)=x[\left.g_{i 1}^{1 y}+\frac{q x}{4} f_{1}^{1 y}+\frac{x^{2}}{40}\left(4 V_{i 0}+q^{2}\right) f_{1}^{1 y}\right] \Theta_{11}(u)+ \\
&+\frac{x^{3}}{10 y^{2}}\left\{y^{2}\left(h_{11}^{y}-V^{00}\right) g_{i 1}^{1 y}-2[\ell(\ell+1)]^{1 / 2} g_{i 0}^{1 y}\right\} \Theta_{11}(u)+ \\
&+x^{2}\left[g_{i 1}^{2 y}+\frac{q x}{6} f_{1}^{2 y}\right] \Theta_{21}(u)+x^{3} g_{i 1}^{3 y} \Theta_{31}(u)+O\left(x^{4}\right), \\
& \Psi_{i 2}^{\varepsilon y}(x, y, \theta)=x^{2}\left[g_{i 2}^{2 y}+\frac{q x}{6} f_{2}^{2 y}\right] \Theta_{22}(u)+x^{3} g_{i 2}^{3 y} \Theta_{32}(u)+O\left(x^{4}\right),
\end{aligned}
$$

где $f_{m^{\prime}}^{n y}(y), g_{i m^{\prime}}^{n y}(y)$ - произвольные функции, но $f_{0}^{n y}, g_{i 0}^{n y} \equiv 0$ при $\sigma=(-1)^{\ell+1}$.

Теперь найдем производные $\partial_{x}^{n}$ полученных асимптотик и, таким образом, выведем связи при $x=0, y>0$ для проекций $\partial_{x}^{n} \Psi_{i b m^{\prime}}^{\varepsilon y} \equiv(n !) \Psi_{i b m^{\prime}}^{n \varepsilon y}$ производных $\partial_{x}^{n} \Psi_{m^{\prime}}^{\varepsilon y}$ на функции $\Theta_{b m^{\prime}}(u)$. При $x=0$ и $q \neq 0$ проекции $\Psi_{i b m^{\prime}}^{\varepsilon y}$ фаддеевской компоненты $\Psi_{i}^{\varepsilon}$ связаны с проекциями $\Psi_{b m^{\prime}}^{\varepsilon y}$ функции $\Psi^{\varepsilon}$ соотношениями

$$
\partial_{x}^{b}\left[2 \partial_{x} \Psi_{i b m^{\prime}}^{\varepsilon y}(x, y)-q \Psi_{b m^{\prime}}^{\varepsilon y}(x, y)\right]=0, \quad b \geqslant m^{\prime}=0,1,2,
$$


а для проекций $\Psi_{i b m^{\prime}}^{\varepsilon y}$ имеются связи

$$
\begin{gathered}
{\left[3 q \partial_{x}^{2}-\left(2 V_{i 0}+q^{2}\right) \partial_{x}-q\left(h_{00}^{y}-V^{00}\right)\right] \Psi_{i 00}^{\varepsilon y}(x, y)=0,} \\
\partial_{x}\left\{12 q \partial_{x}^{2}-\left[q^{3}+2 q\left(4 h_{00}^{y}+3 V_{i 0}-3 V^{00}\right)+12 V_{i 1}\right]\right\} \Psi_{i 00}^{\varepsilon y}(x, y)=0, \\
\partial_{x}\left[10 q \partial_{x}^{2}-3\left(4 V_{i 0}+q^{2}\right) \partial_{x}-6 q\left(h_{10}^{y}-V^{00}\right)\right] \Psi_{i 10}^{\varepsilon y}(x, y)= \\
=-\frac{6 q}{y^{2}}\left[2 \ell(\ell+1)\left(1+\sigma(-1)^{\ell}\right)\right]^{1 / 2} \partial_{x} \Psi_{i 11}^{\varepsilon y}(x, y), \\
\partial_{x}\left[10 q \partial_{x}^{2}-3\left(4 V_{i 0}+q^{2}\right) \partial_{x}-6 q\left(h_{11}^{y}-V^{00}\right)\right] \Psi_{i 11}^{\varepsilon y}(x, y)= \\
=-12 q[\ell(\ell+1)]^{1 / 2} \partial_{x} \Psi_{i 10}^{\varepsilon y}(x, y) .
\end{gathered}
$$

При $q=0$ все эти связи вырождаются в равенства

$$
\left.\partial_{x}^{a+1} \Psi_{i a m^{\prime}}^{\varepsilon y}(x, y)\right|_{x=0}=0, \quad a=0,1,
$$

и, кроме того, имеются связи

$$
\begin{gathered}
{\left[3 \partial_{x}^{2}-\left(h_{00}^{y}-V^{00}\right)\right] \Psi_{i 00}^{\varepsilon}(x, y)=V_{i 0} \Psi_{00}^{\varepsilon y}(x, y),} \\
\left\{2 V_{i 0} \partial_{x}^{3}-V_{i 1}\left[3 \partial_{x}^{2}-\left(h_{00}^{y}-V^{00}\right)\right]\right\} \Psi_{i 00}^{\varepsilon y}(x, y)=0, \\
\partial_{x}\left[5 \partial_{x}^{2}-3\left(h_{10}^{y}-V^{00}\right)\right] \Psi_{i 10}^{\varepsilon y}(x, y)+ \\
+\frac{3}{y^{2}}\left[2 \ell(\ell+1)\left(1+\sigma(-1)^{\ell}\right)\right]^{1 / 2} \partial_{x} \Psi_{i 11}^{\varepsilon y}(x, y)=3 V_{i 0} \partial_{x} \Psi_{10}^{\varepsilon}(x, y), \\
\partial_{x}\left[5 \partial_{x}^{2}-3\left(h_{11}^{y}-V^{00}\right)\right] \Psi_{i 11}^{\varepsilon y}(x, y)+ \\
+\frac{6}{y^{2}}[\ell(\ell+1)]^{1 / 2} \partial_{x} \Psi_{i 10}^{\varepsilon y}(x, y)=3 V_{i 0} \partial_{x} \Psi_{11}^{\varepsilon}(x, y) .
\end{gathered}
$$

Завершим настоящий раздел важными замечаниями.

В работе [10] шестимерные уравнения Фаддеева (3) подстановкой (34) с $t=x$ были впервые редуцированы к конечной системе трехмерных дифференциальных уравнений Фаддеева для совокупности $\left\{\Psi_{j m^{\prime}}^{\varepsilon x}\right\}$ всех искомых $D^{\sigma}$-компонент $\Psi_{j m^{\prime}}^{\varepsilon x}$, $j=1,2,3, m^{\prime} \geqslant \mu(\sigma)$. Теория рассеяния для такой формулировки задачи трех частиц в представлении полного углового момента предложена в работе [11]. После публикации этой работы дискретные сплайн-аналоги трехмерных уравнений Фаддеева стали интенсивно использоваться для расчета характеристик реальных трехчастичных систем. Результаты первых расчетов, выполненных в таком подходе к решению задачи трех частиц с кулоновскими взаимодействиями, обсуждались в обзоре [7]. В случае парных взаимодействий с сильным короткодействующим отталкиванием дискретные сплайн-аналоги трехмерных уравнений Фаддеева впервые построены, исследованы и применены для расчета связанных состояний тримера атома ${ }^{4}$ Не в работах [20], [21]. Затем такие сплайн-аналоги использовались в работе [22], но уже для анализа упругого рассеяния атома ${ }^{4}$ Не на связанном состоянии двух атомов ${ }^{4} \mathrm{He}$.

Как было показано выше, выбор системы $S^{y}$ в качестве “подвижной" является более предпочтительным, потому что при таком выборе фаддеевские $D^{\sigma}$-компоненты 
$\Psi_{i m^{\prime}}^{\varepsilon y}, m^{\prime} \geqslant \mu(\sigma)$, вблизи точки парного удара частиц $p_{j}$ и $p_{k}$ устроены довольно просто. Поэтому для оптимизации численных расчетов предлагается использовать выведенные в работе [23] трехмерные уравнения Фаддеева в случае $t=y$. Доказанные в настоящей работе связи (40)-(42) стоит включать в дискретные сплайн-аналоги таких уравнений для ускорения поточечной сходимости вычисляемых фаддеевских $D^{\sigma}$-компонент $\Psi_{i m^{\prime}}^{\varepsilon y}$ к точным вблизи соответствующих $(i=1,2,3)$ точек парных соударений.

\section{4. СЛУЧАЙ $S$-ВОЛНОВЫХ ВЗАИМОДЕЙСТВИЙ}

Ограничимся случаем трех тождественных частиц. Пусть парные потенциалы $V_{k}\left(x_{k}\right)$ заданы рядами (5), а парные взаимодействия являются $S$-волновыми:

$$
V_{k}\left(\mathbf{x}_{k}\right)=\left|Y_{00}\left(\hat{x}_{k}\right)\right\rangle V_{k}\left(x_{k}\right)\left\langle Y_{00}\left(\hat{x}_{k}\right)\right|, \quad k=1,2,3 .
$$

Как известно [9], в этом случае система уравнений Фаддеева в бисферическом базисе состоит из одного двумерного интегродифференциального уравнения. Его можно вывести разными способами [19]. Для наших целей наиболее удобным является представление фаддеевских компонент $\Psi_{k}^{\varepsilon}$ в виде

$$
\Psi_{k}^{\varepsilon}\left(\mathbf{x}_{k}, \mathbf{y}_{k}\right)=y_{k}^{\ell} G^{\ell}\left(x_{k}, y_{k}\right) \mathcal{Y}_{\ell 0}^{\ell m}\left(\hat{x}_{k}, \hat{y}_{k}\right), \quad k=1,2,3 .
$$

Вследствие тождественности частиц такому представлению отвечает особое бисферическое разложение (17) решения $\Psi^{\varepsilon}$ уравнения Шредингера (2), в котором при $b=1,2, \ldots \Psi_{a b}^{\varepsilon} \equiv 0$, а при $b \neq 1,2, \ldots$

$$
\Psi_{a b}^{\varepsilon}(x, y)=y^{\ell} G^{\ell}(x, y) \delta_{a \ell} \delta_{b 0}+2 \int_{-1}^{1} K_{a b \ell 0}^{\ell}(x, y, u)\left(y^{\prime}\right)^{\ell} G^{\ell}\left(x^{\prime}, y^{\prime}\right) d u
$$

Здесь и далее $G^{\ell}\left(x_{k}, y_{k}\right)$ - искомая функция; $x \equiv x_{i}, y \equiv y_{i}$ - аргументы; переменные $x^{\prime} \equiv x_{k}, y^{\prime} \equiv y_{k}-$ функции (10) или (11) аргументов $x, y, u$ при $\gamma_{k i}=\pi / 3$; а ядра $K_{a b \ell 0}^{\ell}\left(y^{\prime}\right)^{\ell}-$ функции, исследованные в работах [18], [19].

В общем случае эти функции - конечные двойные суммы:

$$
\begin{aligned}
& K_{a b \ell 0}^{\ell}(x, y, u)\left(y^{\prime}\right)^{\ell}= y^{\ell} \sum_{n=0}^{\ell}\left(\frac{x}{y}\right)^{n} \sum_{s=b-n}^{b+n} K_{a b}^{\ell n s} P_{s}(u), \quad(-1)^{n+s+b}=1, \\
& K_{a b}^{\ell n s} \equiv(-1)^{a}(\sqrt{3})^{\ell-n}\left(\frac{1}{2}\right)^{\ell}\left[\frac{(2 \ell+1) !}{(2 \ell-2 n) ! n !}\right]^{1 / 2} \times \\
& \times(2 s+1) C_{s 0 \ell-n 0}^{a 0} C_{s 0 n 0}^{b 0}\left\{\begin{array}{ccc}
a & b & \ell \\
n & \ell-n & s
\end{array}\right\},
\end{aligned}
$$

а в случае $a=\ell, b=0$ - однократные суммы:

$$
\begin{gathered}
K_{\ell 0 \ell 0}^{\ell}(x, y, u)\left(y^{\prime}\right)^{\ell}=y^{\ell} \sum_{n=0}^{\ell}\left(\frac{x}{y}\right)^{n} K^{\ell n} P_{n}(u), \\
K^{\ell n} \equiv \frac{1}{2}(-1)^{\ell}\left(\frac{1}{2}\right)^{\ell} \frac{(\sqrt{3})^{n} \ell !}{n !(\ell-n) !} .
\end{gathered}
$$


Заменим в системе Фаддеева (3) функции $\Psi_{k}^{\varepsilon}$ и $\Psi^{\varepsilon}$ их бисферическими рядами с компонентами (43), (44). Спроецируем полученные уравнения на бисферический базис и в результате для искомой функций $G^{\ell}$ выведем двумерное интегродифференциальное уравнение с ядром $K_{\ell 0 \ell 0}^{\ell}\left(y^{\prime}\right)^{\ell}$. Заменив это ядро суммой $(46)$, получаем уравнение

$$
\begin{aligned}
\left\{\partial_{x}^{2}\right. & \left.+\frac{2}{x} \partial_{x}+\partial_{y}^{2}+(\ell+1) \frac{2}{y} \partial_{y}+E-V_{i}(x)\right\} G^{\ell}(x, y)= \\
& =2 V_{i}(x) \sum_{n=0}^{\ell}\left[K^{\ell n}\left(\frac{x}{y}\right)^{n} \int_{-1}^{1} d u P_{n}(u) G^{\ell}\left(x^{\prime}, y^{\prime}\right)\right] .
\end{aligned}
$$

Построим решение $G^{\ell}$ этого уравнения в виде формального ряда

$$
G^{\ell}(x, y)=\sum_{n=0}^{\infty} x^{n} G_{n}^{\ell}(y), \quad G_{n}^{\ell}(y)=\left.\frac{1}{n !} \partial_{x}^{n} G^{\ell}(x, y)\right|_{x=0} .
$$

Начнем с анализа ряда Тейлора с центром в точке $x=0$ для функции $G^{\ell}\left(x^{\prime}, y^{\prime}\right)$, стоящей под знаком интеграла. Ее аргументы $x^{\prime}$ и $y^{\prime}-$ функции вида (11) переменных $x, y, u$ при $\gamma=\pi / 3$, поэтому производные $\partial_{x}^{n} G^{\ell}$ - сложные комбинации производных $\partial_{x}^{p} x^{\prime}, \partial_{x}^{p} y^{\prime}$ и их целых степеней, например

$$
\begin{aligned}
& \partial_{x} G^{\ell}\left(x^{\prime}, y^{\prime}\right)=\left[\left(\partial_{x} x^{\prime}\right) \partial_{x^{\prime}}+\left(\partial_{x} y^{\prime}\right) \partial_{y^{\prime}}\right] G^{\ell}\left(x^{\prime}, y^{\prime}\right), \\
& \partial_{x}^{2} G^{\ell}\left(x^{\prime}, y^{\prime}\right)=\left\{\left[\left(\partial_{x} x^{\prime}\right) \partial_{x^{\prime}}+\left(\partial_{x} y^{\prime}\right) \partial_{y^{\prime}}\right]^{2}+\left(\partial_{x} x^{\prime}\right)^{2} \partial_{x^{\prime}}+\left(\partial_{x} y^{\prime}\right)^{2} \partial_{y^{\prime}}\right\} G^{\ell}\left(x^{\prime}, y^{\prime}\right) .
\end{aligned}
$$

В силу (13) и (14) производные $\partial_{x}^{p} x^{\prime}$ и $\partial_{x}^{p} y^{\prime}$ - конечные суммы по полиномам Лежандра. Поэтому исследуемый ряд Тейлора функции $G^{\ell}$ - двойная сумма

$$
G^{\ell}\left(x^{\prime}, y^{\prime}\right)=\sum_{p=0}^{\infty} x^{p} \sum_{s=0}^{p} G_{p s}^{\ell}(y) P_{s}(u), \quad(-1)^{p+s}=1,
$$

в которой функции $G_{p s}^{\ell}(y)$ - довольно громоздкие комбинации производных функции $G^{\ell}\left(x^{\prime}, y^{\prime}\right)$ при $x=0$, т. е. в точке $\left(x^{\prime}, y^{\prime}\right)=(\sqrt{3} y / 2, y / 2)$. Например,

$$
\begin{array}{cc}
G_{00}^{\ell}(y)=G^{\ell}\left(x^{\prime}, y^{\prime}\right), & G_{11}^{\ell}(y)=\frac{1}{2}\left(\partial_{x^{\prime}}-\sqrt{3} \partial_{y^{\prime}}\right) G^{\ell}\left(x^{\prime}, y^{\prime}\right), \\
G_{20}^{\ell}(y)=\left(d_{2}+d_{1}\right) G^{\ell}\left(x^{\prime}, y^{\prime}\right), & G_{22}^{\ell}(y)=\left(2 d_{2}-d_{1}\right) G^{\ell}\left(x^{\prime}, y^{\prime}\right), \\
d_{1} \equiv \frac{1}{6 \sqrt{3}}\left(\partial_{x^{\prime}}+3 \sqrt{3} \partial_{y^{\prime}}\right), & d_{2} \equiv \frac{1}{24}\left(\partial_{x^{\prime}}-\sqrt{3} \partial_{y^{\prime}}\right)^{2} .
\end{array}
$$

Докажем правило отбора $(-1)^{p+s}=1$ для ряда (49). Как следует из (10), функции $x^{\prime}$ и $y^{\prime}$ инвариантны относительно одновременной инверсии $x \rightarrow-x, u \rightarrow-u$. Значит любая функция $G^{\ell}\left(x^{\prime}, y^{\prime}\right)$ и ее разложение (49) обладают этим же свойством. Каждое слагаемое $x^{s} G_{p s}(y) P_{s}(u)$ такого ряда при инверсии приобретает множитель $(-1)^{p+s}$, а все слагаемые линейно независимы по одному или двум аргументам $x$ и $u$. Поэтому исследуемый ряд инвариантен относительно инверсии тогда и только 
тогда, когда его индексы $p$ и $s$ такие, что $p+s$ - четное число, что и требовалось показать.

Теперь в уравнении (47) заменим функции $V_{i}(x), G^{\ell}(x, y)$ и $G^{\ell}\left(x^{\prime}, y^{\prime}\right)$ соответствующими рядами (5), (48) и (49). Из получившегося уравнения для искомых функций $G_{n}^{\ell}$ выведем рекуррентную цепочку уравнений

$$
\begin{aligned}
(n+2)(n+3) G_{n+2}^{\ell}(y) & =q G_{n+1}^{\ell}(y)+\tilde{h}_{\ell}(y) G_{n}^{\ell}(y)+ \\
+ & \sum_{p=1}^{n} V_{i p} G_{n-p}^{\ell}(y)+\sum_{p=0}^{n} \sum_{s=0}^{\min \{\ell, p\}} V_{i, n-p-s} \frac{4 K^{\ell s}}{2 s+1} y^{-s} G_{p s}^{\ell}(y),
\end{aligned}
$$

где $n=-2,-1, \ldots$, а $G_{n}^{\ell} \equiv 0$ при $n<0$ и по определению

$$
\tilde{h}_{\ell}(y) \equiv-\partial_{y}^{2}-(\ell+1) \frac{2}{y} \partial_{y}+V_{i 0}-E .
$$

Все функции $G_{n}^{\ell}(y)$, удовлетворяющие этой цепочке, выражаются явно через неопределенную функцию $G_{0}^{\ell}(y)=G^{\ell}(0, y)$ и функции $G_{p s}^{\ell}(y)$. Заменим этими выражениями функции $G_{n}^{\ell}(y), n \leqslant 3$, в сумме $(48)$, а затем запишем ее асимптотику при $x \rightarrow 0$, $y>0$ в виде

$$
\begin{aligned}
G^{\ell}(x, y)=\{1 & +\frac{q x}{2}+\frac{x^{2}}{12}\left(2 \tilde{h}_{\ell}(y)+q^{2}\right)+ \\
& \left.+\frac{x^{3}}{144}\left[q\left(8 \tilde{h}_{\ell}(y)+q^{2}\right)+12 V_{i 1}\right]\right\} G^{\ell}(0, y)+ \\
+ & x K^{\ell 0}\left\{2 q+\frac{x}{3}\left(q^{2}+2 V_{i 0}\right)+\frac{x^{2}}{36}\left[q\left(q^{2}+6 \tilde{h}_{\ell}(y)+2 V_{i 0}\right)+12 V_{i 1}\right]\right\} G_{00}^{\ell}(y)+ \\
& +x^{3} \frac{q}{9} K^{\ell 0}\left[-\frac{\ell \sqrt{3}}{y} G_{11}^{\ell}(y)+3 G_{20}^{\ell}(y)\right]+O\left(x^{4}\right)
\end{aligned}
$$

где $K^{\ell s}$ и $G_{00}^{\ell}$ определены формулами (46) и (50). Из этой асимптотики в случае $q \neq 0$ находим следующие связи при $x=0, y>0$ и $\left(x^{\prime}, y^{\prime}\right)=(\sqrt{3} y / 2, y / 2)$ :

$$
\begin{gathered}
\left(2 \partial_{x}-q\right) G^{\ell}(x, y)=(-1)^{\ell} 2^{1-\ell} q G^{\ell}\left(x^{\prime}, y^{\prime}\right), \\
{\left[3 q \partial_{x}^{2}-\left(2 V_{i 0}+q^{2}\right) \partial_{x}-q\left(\tilde{h}_{\ell}+V_{i 0}\right)\right] G^{\ell}(x, y)=0,} \\
\left\{12 q \partial_{x}^{3}-\left[q\left(6 \tilde{h}_{\ell}+2 V_{i 0}+q^{2}\right)+12 V_{i 1}\right] \partial_{x}-q^{2}\left(\tilde{h}_{\ell}+V_{i 0}\right)\right\} G^{\ell}(x, y)= \\
=(-1)^{\ell} 2^{-\ell-1} q^{2}\left\{\left(\partial_{x^{\prime}}-\sqrt{3} \partial_{y^{\prime}}\right)^{2}+\right. \\
\left.+\frac{4}{\sqrt{3}} y^{-1}\left[(1-3 \ell) \partial_{x^{\prime}}+3(1+3 \ell) \partial_{y^{\prime}}\right]\right\} G^{\ell}\left(x^{\prime}, y^{\prime}\right),
\end{gathered}
$$

а при $q=0$ получаем равенство $\left.\partial_{x} G^{\ell}(x, y)\right|_{x=0}=0$ и связи

$$
\begin{gathered}
{\left[3 \partial_{x}-\tilde{h}_{\ell}(y)\right] G^{\ell}(x, y)=(-1)^{\ell} 2^{1-\ell} G^{\ell}\left(x^{\prime}, y^{\prime}\right),} \\
\left\{2 V_{i 0} \partial_{x}^{3}-V_{i 1}\left[3 \partial_{x}^{2}+V_{i 0}-\tilde{h}_{\ell}(y)\right]\right\} G^{\ell}(x, y)=0 .
\end{gathered}
$$


В полученной серии связей (53), (54) имеются особые связи, содержащие функцию $G^{\ell}\left(x^{\prime}, y^{\prime}\right)$. Эти соотношения связывают значения исследуемой функции $G^{\ell}(x, y)$ на прямой $(x=0, y>0)$ с ее значениями, но на прямой $y=\sqrt{3} x>0$. Такая связь является следствием нелокальности уравнения (47) и означает, что поведение фаддеевской бисферической компоненты $\Psi_{i \ell 0}^{\varepsilon}=y^{\ell} G^{\ell}(x, y)$ в точке парного удара $(x=0, y>0)$ зависит и от значений ее частных производных в точке $(\sqrt{3} y / 2, y / 2)$.

Теперь исследуем строение бисферических компонент $\Psi_{a b}^{\varepsilon}$ разложения (17) решения $\Psi^{\varepsilon}$ уравнения Шредингера (2), отвечающее найденному разложению (48) функции $G^{\ell}$. Заменим в представлениях (44) этих компонент функции $G^{\ell}(x, y)$ и $G^{\ell}\left(x^{\prime}, y^{\prime}\right)$ рядами (48) и (49), а ядро $K_{a b \ell 0}^{\ell}\left(y^{\prime}\right)^{\ell}$ - суммой (45). Рассмотрим получившийся ряд

$$
\Psi_{a b}^{\varepsilon}(x, y)=y^{\ell} \sum_{n=0}^{\infty} x^{n} G_{n}^{\ell}(y) \delta_{a \ell} \delta_{b 0}+y^{\ell} \sum_{m=0}^{\infty} x^{m} \sum_{p=m-\ell}^{m} y^{p-m} \sum_{s=b-m+p}^{b+m-p} \frac{4 K_{a b}^{\ell, m-p, s}}{2 s+1} G_{p s}^{\ell}(y),
$$

где $b$ - четное из-за тождественности частиц, а обе суммы $p+s$ и $m-p+s-$ четные в силу правил отбора для рядов (45) и (49). Поэтому $m$ четно. Следовательно, вблизи точки парного удара компонента $\Psi_{\ell 0}^{\varepsilon}(x, y)$ - ряд по четным и нечетным степеням аргумента $x$, а все остальные компоненты $\Psi_{a b}^{\varepsilon}(x, y), b=2,4, \ldots,-$ ряды по четным степеням этого же аргумента.

Явные асимптотики компонент $\Psi_{a b}^{\varepsilon}(x, y)$ при $x \rightarrow 0$ несложно найти как подсуммы наиболее медленно убывающих слагаемых рядов (55).

Например, при $b=0$

$$
\begin{aligned}
\Psi_{\ell 0}^{\ell}(x, y)=y^{\ell} & \left(1+\frac{q x}{2}\right)\left[G_{0}^{\ell}(y)+2\left(-\frac{1}{2}\right)^{\ell} G_{00}^{\ell}(y)\right]- \\
& -x^{2} \frac{2}{\sqrt{3}}\left(-\frac{y}{2}\right)^{\ell-1} C_{10 \ell-10}^{\ell 0} G_{11}^{\ell}(y)+O\left(x^{3}\right)
\end{aligned}
$$

при $b=2$

$$
\begin{aligned}
\Psi_{a 2}^{\varepsilon}=x^{2} & \frac{2}{\sqrt{5}}\left(-\frac{y}{2}\right)^{\ell}\left\{C_{\ell 020}^{a 0} G_{22}^{\ell}(y)+\right. \\
& +\frac{1}{y \sqrt{3}}[(\ell+a+2)(\ell+a+3)(\ell-a+2)(\ell-a+1)]^{1 / 2} C_{10 \ell-10}^{a 0} G_{11}^{\ell}(y)+ \\
& \left.+3 y^{-2}[(2 \ell+1)(2 \ell-1) \ell(\ell-1)]^{1 / 2} G_{00}^{\ell}(y) \delta_{a, \ell-2}\right\}+O\left(x^{4}\right)
\end{aligned}
$$

С учетом представлений (17), (56), (57) исследуемое решение $\Psi^{\varepsilon}$ при $x \rightarrow 0$ и $y>0$ обладает следующим свойством:

$$
\Psi^{\varepsilon}(\mathbf{x}, \mathbf{y})=\Psi_{\ell 0}^{\varepsilon}(x, y) \mathcal{Y}_{\ell 0}^{\ell m}(\hat{x}, \hat{y})[1+o(1)]=\left.\left(1+\frac{q x}{2}\right) \Psi^{\varepsilon}(\mathbf{x}, \mathbf{y})\right|_{x=0}+O\left(x^{2}\right),
$$

поэтому подчиняется связи

$$
\left(2-\partial_{x}-q\right) \Psi^{\varepsilon}(x, y)=0, \quad x=0, \quad y>0 .
$$


При $q \neq 0$ и $\ell=0$ эта связь, выведенная нами для случая $S$-волновых взаимодействий, является аналогом условия Като, доказанного в работе [1], но в случае центральных, а именно кулоновских, парных взаимодействий.

\section{5. МЕТОДИЧЕСКИЕ ЗАМЕЧАНИЯ}

В разделе 3 показано, как построить разложения одной фаддеевской компоненты $\Psi_{i}^{\varepsilon}$ и ее парциальных компонент $\Psi_{i a b}^{\varepsilon}$ и $\Psi_{i m^{\prime}}^{\varepsilon y}$ вблизи точки парного удара двух выбранных частиц $p_{j}$ и $p_{k}$. Полагая последовательно $i=1,2,3$ и повторяя каждый раз все построения, нетрудно найти разложения всех парциальных компонент $\Psi_{i a b}^{\varepsilon}$ и $\Psi_{i m^{\prime}}^{\varepsilon y}, i=1,2,3$, вблизи точек столкновения соответствующих частиц $p_{j}$ и $p_{k}$. Таким образом, в случае центральных парных взаимодействий завершается построение разложений решений $\left\{\Psi_{j a b}^{\varepsilon}\right\}$ и $\left\{\Psi_{j m^{\prime}}^{\varepsilon y}\right\}$ двумерных и трехмерных уравнений Фаддеева вблизи любой из трех точек парных ударов.

Предложенный в разделе 3 метод является достаточно общим по двум причинам. Во-первых, этот метод применим для системы трех разных частиц с центральными парными взаимодействиями довольно общего для задач атомной и молекулярной физики типа (5) и при любых допустимых значениях полного углового момента $\ell$ и пространственной четности $\sigma$. Во-вторых, этим методом можно исследовать полные (бесконечные) формальные разложения регулярных решений двумерных и трехмерных уравнений Фаддеева в малых окрестностях всех трех точек парных соударений.

Главное преимущество предложенного метода - его исключительная простота, заключающаяся в том, что построение разложений всех исследуемых функций сводится к явному решению соответствующих рекуррентных цепочек алгебраических уравнений.

Метод реализуется в три этапа и начинается с уравнения Шредингера (2). Сначала бисферические или $D^{\sigma}$-компоненты $\Psi_{a b}^{n \varepsilon}$ или $\Psi_{m^{\prime}}^{n \varepsilon t}$ решения $\Psi^{\varepsilon}$ этого уравнения строятся в виде рядов (18) или (21) способами, предложенными в работе [8]. Затем в уравнении системы Фаддеева (3), содержащем в левой части компоненту $\Psi_{i}^{\varepsilon}$, функция $\Psi^{\varepsilon}$ заменяется одним из таких рядов, взаимодействие $V_{i}$ представляется известным рядом (5), а компонента $\Psi_{i}^{\varepsilon}$ восстанавливается как соответствующий выбранному разложению функции $\Psi^{\varepsilon}$ ряд (22), (23) или (36), (37) с искомыми функциями $\Psi_{i a b}^{n \varepsilon}(y)$ или $\Psi_{i m^{\prime}}^{n \varepsilon y}(y)$. Наконец, эти функции выражаются как решения рекуррентных цепочек уравнений (25) или (38) через известные функции $\Psi_{a b}^{n \varepsilon}$ или $\Psi_{m^{\prime}}^{n \varepsilon t}$.

Все вышеупомянутые этапы реализуются исключительно просто благодаря тому, что для анализа уравнения Шредингера и лишь одного из уравнений системы Фаддеева используется только собственное для этих уравнений координатное представление $\langle\mathbf{x}, \mathbf{y}| \equiv\left\langle\mathbf{x}_{i}, \mathbf{y}_{i}\right|$.

Альтернативный нашему метод построения разложений фаддеевских парциальных компонент в точке парного удара впервые был предложен в работе [10], где решение $\Psi^{\varepsilon}$ уравнения Шредингера не используется, а основными являются одновременно все три шестимерных уравнения Фаддеева (3) и разложения всех $(k=1,2,3)$ парных взаимодействий $V_{k}\left(x_{k}\right)$ и всех искомых компонент $\Psi_{k}^{\varepsilon}$ в ряды Тейлора с 
центром в точке $x=x_{i}=0$ :

$$
\Psi_{k}^{\varepsilon}\left(\mathbf{x}_{k}, \mathbf{y}_{k}\right)=\sum_{n=0}^{\infty} x^{n} \Psi_{k}^{n \varepsilon}(\hat{x}, \mathbf{y}), \quad \Psi_{k}^{n}(\hat{x}, \mathbf{y})=\left.\frac{1}{n !} \partial_{x}^{n} \Psi_{k}^{\varepsilon}\left(\mathbf{x}_{k}, \mathbf{y}_{k}\right)\right|_{x=0} .
$$

Переменные $x_{k}$ и $y_{k}$ являются функциями (10) аргументов $x$ и $y$, поэтому уже при $n=3$ производные $\partial_{x}^{n} \Psi_{k}^{\varepsilon}\left(\mathbf{x}_{k}, \mathbf{y}_{k}\right)$ - довольно сложные комбинации производных $\partial_{x}^{p} x_{k}$ и $\partial_{x}^{p} y_{k}$ и производных функции $\Psi_{k}^{\varepsilon}$ по аргументам $x_{k}$ и $y_{k}$. Так как функция $\Psi^{\varepsilon}$ считается неизвестной, приходится решать все три уравнения Фаддеева и выводить одновременно разложения при $x_{i} \rightarrow 0$ и $y_{i}>0$ для всех трех компонент $\Psi_{i}^{\varepsilon}$ и $\Psi_{k}^{\varepsilon}$, $k \neq i$. Решение существенно усложняется тем, что искомые разложения необходимо записывать в каждом из трех координатных представлений $\left\langle\mathbf{x}_{k}, \mathbf{y}_{k}\right|, k=1,2,3$. По той же причине сложным оказывается и решение заключительной задачи восстановления разложений функции $\Psi^{\varepsilon}$ по найденным разложениям ее фаддеевских компонент $\Psi_{i}^{\varepsilon}$.

В случае центральных парных взаимодействий наш подход более экономичен и прост по сравнению с альтернативным подходом работы [10].

Если же парные взаимодействия нецентральные, то в бисферическом базисе система уравнений Шредингера бесконечная, а система уравнений Фаддеева конечная. Поэтому выгоднее сначала вывести разложения бисферических фаддеевских компонент $\Psi_{i a b}^{\varepsilon}$, т. е. применить метод обсужденной выше работы [10], а затем по найденным представлениям воспроизвести разложения компонент $\Psi_{i}^{\varepsilon}$ и их суммы $\Psi^{\varepsilon}$, подчиненной уравнению Шредингера. Именно по такой схеме выполнены исследования, представленные в разделе 4.

\section{6. ЗАКЛЮЧЕНИЕ}

Суммируем основные результаты настоящей работы.

Было доказано, что если все центральные парные взаимодействия - ряды (5), то при $x \rightarrow 0$ и $y>0$ представлениям (17) и (19) решения $\Psi^{\varepsilon}(\mathbf{x}, \mathbf{y})$ уравнения Шредингера (2) соответствуют аналогичные по строению разложения (22), (23) и (34)-(37) фаддеевской компоненты $\Psi_{i}^{\varepsilon}(\mathbf{x}, \mathbf{y})$ этого решения. Компоненты $\Psi_{i a b}^{n \varepsilon}$ и $\Psi_{i b m^{\prime}}^{n \varepsilon y}$ этих разложений подчинены разрешимым в явном виде рекуррентным цепочкам алгебраических уравнений $(25)$ и (38).

Предложенная редукция исходных шестимерных уравнений Фаддеева (3) к таким цепочкам позволила вывести явные асимптотики (27)-(29) и (39) бисферических $\Psi_{i a b}^{\varepsilon}$ и $D^{\sigma}$-компонент $\Psi_{i m^{\prime}}^{\varepsilon y}$ физического решения $\Psi_{i}^{\varepsilon}$ вплоть до слагаемых $O\left(x^{4}\right)$ и $O\left(x^{3}\right)$. Из этих асимптотик выведены ранее неизвестные связи (30)-(33) и $(40)-(42)$.

В случае трех тождественных частиц и $S$-волновых взаимодействий доказано следующее утверждение: если потенциалы таких взаимодействий являются степенными рядами (5), то при $x \rightarrow 0$ и $y>0$ формальным решением $G^{\ell}(x, y)$ уравнения (47) является ряд (49) по целым степеням $x$ с компонентами $G_{n}^{\ell}(y)$, подчиненными рекуррентной и разрешимой в явном виде цепочке уравнений (51). Для функции $G^{\ell}$ получена явная асимптотика (52) и связи (53), (54) в точке парного удара. Показано, что

5 Теоретическая и математическая физика, т. 156, № 1, 2008 г. 
такой асимптотике отвечают асимптотики (56), (57) бисферических компонент $\Psi_{a b}^{\varepsilon}$ решения $\Psi^{\varepsilon}$ уравнения Шредингера.

\section{Список литературы}

[1] T. Kato, Comm. Pure Appl. Math., 10:2 (1957), 151-177.

[2] W. A. Bingel, Z. Naturforsch. A, 18 (1963), 1249-1253.

[3] R. T. Pack, W. B. Brown, J. Chem. Phys., 45 (1966), 556-559.

[4] С. И. Виницкий, Л. И. Пономарев, ЭЧАЯ, 13:6 (1982), 1336-1418.

[5] В. И. Коробов, ЯФ, 50:6 (1989), 1595-1604.

[6] V. I. Korobov, I. V. Puzynin, S. I. Vinitsky, Muon Catalyzed Fusion, 7 (1992), 63-80.

[7] В. В. Пупышев, ЭЧАЯ, 33:4 (2002), 843-914.

[8] В. В. Пупышев, ТМФ, 136:1 (2003), 90-114.

[9] С. П. Меркурьев, Л. Д. Фаддеев, Квантовая теория рассеяния для систем нескольких частии, Наука, М., 1985.

[10] V. V. Kostrykin, A. A. Kvitsinsky, S. P. Merkuriev, Few-Body Systems, 6:2 (1989), 97-113.

[11] А. А. Квицинский, В. В. Кострыкин, С. П. Меркурьев, ЭЧАЯ, 21:6 (1990), 1301-1359.

[12] В. В. Пупышев, ЭЧАЯ, 35:2 (2004), 256-347.

[13] В. В. Пупышев, Писъма в ЭЧАЯ, 3:2 (2006), 28-42.

[14] М. В. Федорюк, Асимптотические методы для линейных обыкновенных дифференииальных уравнений, Наука, М., 1983.

[15] А. М. Ильин, Согласование асимтотических разложений решений краевых задач, Наука, М., 1989.

[16] Д. А. Варшалович, А. Н. Москалев, В. К. Херсонский, Квантовая теория углового момента, Наука, М., 1975.

[17] F. T. Smith, J. Chem. Phys., 31:5 (1959), 1352-1359.

[18] В. В. Пупышев, ТМФ, 81:1 (1989), 86-93.

[19] В. В. Пупышев, ЭЧАЯ, 28:6 (1999), 1561-1649.

[20] V. A. Roudnev, S. L. Yakovlev, Comput. Phys. Comm., 126:1-2 (2000), 162-164.

[21] V. A. Roudnev, S. L. Yakovlev, Chem. Phys. Lett., 328:1-2 (2000), 97-106.

[22] V. A. Roudnev, Chem. Phys. Lett., 367:1-2 (2003), 95-101.

[23] В. В. Пупышев, ТМФ, 148:2 (2006), 227-242.

Поступила в редакцию 29.03.2007 